The Journal of Rheumatology 2022;xx:xxxx

doi: $10.3899 /$ jrheum. 211190

First Release December 152021

\section{Dr. Sehgal et al reply}

\section{To the Editor:}

We appreciate the interest of Wang et $\mathrm{al}^{1}$ in our case. ${ }^{2}$ We agree with several of their comments and would like to clarify a few details here.

While magnetic resonance imaging (MRI) is helpful in enhancing diagnostic capabilities in eosinophilic fasciitis (EF), ${ }^{3}$ full-thickness skin biopsy is considered the gold standard for EF diagnosis. Ultrasound is an inexpensive, readily available modality in rheumatology practices in the United States and its role in the diagnosis of EF was reported previously. ${ }^{4}$ Our patient's joint and limb contractures, the difficulty in achieving optimal positioning in MRI scanner, and cost and convenience favored the use of ultrasound instead of MRI as an imaging modality to follow EF activity in our case.

We agree that there remains an unmet need for assessing disease activity in fibrosing disorders such as EF. Although range of motion as measured by a physical therapist has been used in a previous case report using tofacitinib in $\mathrm{EF}^{5}$ the authors acknowledge that our assessment was clinical and not quantified, and this may be a limitation of our case.

Monitoring disease activity in EF is challenging since no standardized outcome measures have been validated. ${ }^{6} \mathrm{EF}$ is included in the spectrum of scleroderma-like disorders, ${ }^{7}$ and while modified Rodnan skin score (mRSS) is useful for systemic sclerosis, its use in scleroderma spectrum disorders has not been validated. We observed improvement in both mRSS (prebaricitinib: 31; postbaricitinib: 14) and modified Health Assessment Questionnaire (mHAQ) scores for our patient, although only the mHAQ was included in this report for reasons mentioned earlier. Peripheral blood eosinophilia improvement and normalization was observed on systemic corticosteroids and other immunomodulatory therapies even prior to baricitinib use, although it did not seem to translate to improvement in joint mobility, skin induration, edema, and elasticity. We agree with the comments of Wang et $\mathrm{al}^{1}$ on the mechanism of action of Janus kinase (JAK) inhibitors, which were omitted in the interest of case description, therapy, and outcome.

Baricitinib is available only in 2-mg doses in the US; this was the dose used in our patient for 6 months. The US Food and Drug Administration did not approve the 4-mg dose (available in Europe and several other countries), citing safety concerns. We did not observe any safety concerns with its use in our patient, including cytopenias, infections, or thrombosis for the duration of treatment.

Our patient had a recent 6-month follow-up after completion of treatment with baricitinib without any relapse of fasciitis or peripheral blood eosinophilia.

The authors would like to emphasize that this is 1 case report and we are certainly in need of large-scale, randomized, placebo-controlled studies to confirm the efficacy of JAK inhibitors in treating EF. However, given the rarity of this disease, it is challenging to conduct these kinds of trials.

Rahul Sehgal ${ }^{1}(D$, Assistant Professor of Medicine, MBBS, MD Floranne C. Ernste ${ }^{1}$ (I), Associate Professor of Medicine, MD Sara Eckloff' ${ }^{1}$, Assistant Professor of Medicine, MD ${ }^{1}$ Department of Medicine, Division of Rheumatology, Mayo Clinic, Rochester, Minnesota, USA.

R. Sehgal passed away on November 10, 2021.

The authors declare no conflicts of interest relevant to this article.

Address correspondence to Dr. F. Ernste, Mayo Clinic, 200 First Street SW, Rochester, MN 55905, USA. Email: ernste.floranne@mayo.edu.

\section{REFERENCES}

1. Wang G, Zhuo N, Li J. Baricitinib for refractory eosinophilic fasciitis: myth or reality? J Rheumatol xxxxxx.

2. Sehgal R, Ernste FC, Eckloff S. Successful treatment with baricitinib in a patient with refractory eosinophilic fasciitis. J Rheumatol 2021;48:948-9.

3. Moulton SJ, Kransdorf MJ, Ginsburg WW, Abril A, Presllin S. Eosinophilic fasciitis: spectrum of MRI findings. AJR Am J Roentgenol 2005; 184:975-8.

4. Mondal S, Goswami RP, Sinha D, Ghosh A. Ultrasound is a useful adjunct in diagnosis of eosinophilic fasciitis. Rheumatology 2015;54:2041.

5. Kim SR, Charos A, Damsky W, Heald P, Girardi M, King BA. Treatment of generalized deep morphea and eosinophilic fasciitis with the Janus kinase inhibitor tofacitinib. JAAD Case Rep 2018;4:443-5.

6. Fett N, Arthur M. Eosinophilic fasciitis: current concepts. Clin Dermatol 2018;36:487-97.

7. Mori Y, Kahari VM, Varga J. Scleroderma-like cutaneous syndromes. Curr Rheumatol Rep 2002;4:113-22. 